\title{
The Curci-Ferrari model with massive quarks at two loops
}

\author{
R.E. Browne \& J.A. Gracey, \\ Theoretical Physics Division, \\ Department of Mathematical Sciences, \\ University of Liverpool, \\ Peach Street, \\ Liverpool, \\ L69 7ZF, \\ United Kingdom.
}

\begin{abstract}
Massive quarks are included in the Curci-Ferrari model and the theory is renormalized at two loops in the $\overline{\mathrm{MS}}$ scheme in an arbitrary covariant gauge.
\end{abstract}


Recently there has been renewed interest in examining covariantly gauge fixed QCD where the gluon and ghost fields are given explicit mass terms. For instance, in [1, 2, 3] such models have been used to investigate how a mass gap emerges for the gluon. In particular a dimension two operator obtains a non-zero vacuum expectation value which generates a gluon mass. Indeed in this context the effective potential calculation of [3] demonstrated that the non-perturbative vacuum favoured a non-zero vacuum expectation value for the simplest dimension two operator possible in Yang-Mills theories. In the main these studies did not involve quarks and were effectively based on a version of Yang-Mills theory with massive gluons originally introduced by Curci and Ferrari in [4]. There a renormalizable theory of massive gluons was constructed with the aim of being an alternative to the Higgs mechanism for endowing vector bosons with mass. The main shortcoming of the Curci-Ferrari model, however, was the breaking of unitarity directly as a result of the massive gluon, [5, 6, 0, 8, 9, 10]. Nevertheless the model has proved useful for a variety of reasons. From a field theoretic point of view it is of interest due to the nonlinear nature of the gauge fixing term which introduces a quartic ghost self-interaction as well as modifying the usual ghost gluon interaction present in a linear covariant gauge fixing, [4]. The nonlinear property has been examined in [10, 11]. Moreover, there has been a debate on the BRST symmetry of the Curci-Ferrari model. For instance, Ojima, [6], has carried out a comprehensive examination of the BRST algebra with and without a mass term and explicitly constructed a negative norm state which therefore supports the lack of unitarity in the model. Other such states have been determined in [8]. Another feature which emerged in these papers was the non-nilpotency of the BRST charge which appears to follow as a consequence of the exact form of the Lagrangian. A more recent study of this has been given in [12]. A final motivation for considering massive gluons rests in phenomenological considerations. For example, lattice results, [13], suggest that at low energies the gluon is massive though the precise form of the propagator at this scale is not known explicitly but is clearly dependent on non-perturbative properties. However, one can use a Curci-Ferrari type theory with the hope that it can provide useful insight into physics which is in someway dependent on a gluon which is massive. For instance, diffractive scattering has been examined in [14] with such a motivation.

Whilst the majority of the papers in this area concentrate on Yang-Mills theory with a massive gluon the real world is based on QCD which involves quarks. Therefore, if complete studies of the property of a massive gluon are to be performed, massive quarks need to be included. In [6] matter fields were considered but were shown not to affect the failings of the Curci-Ferrari model such as lack of unitarity or non-nilpotency of the BRST charge. The multiplicative renormalizabilty of the model, which was proved in [4, 8, 9, 10], was unaffected. Given that the Curci-Ferrari model has been renormalized at one loop in [8, 12, 15] and more recently at two loops in $\overline{\mathrm{MS}}$ in [16], the purpose of this letter is to extend the renormalization of the Curci-Ferrari model at two loops to the case where massive quarks are included. This is important for various reasons. First, the main motivation of [16] was to provide a calculational tool for renormalizing Green's functions of Yang-Mills theories where one could not naively nullify the momenta of several external legs without introducing spurious infrared infinities. Ordinarily one can handle such infrared problems if some form of infrared rearrangement, [15, 16], is performed to eliminate those divergences which cannot be distinguished from ultraviolet ones in a dimensionally regularized calculation. Moreover, given that infrared rearrangement is usually carried out by hand it does not lend itself readily to automatic calculations by computer. One simple resolution of the infrared problem is to introduce an infrared mass regularization. However, in using the Curci-Ferrari model, [4], which naturally incorporates a gluon and ghost mass and preserves renormalizability one resolves the infrared problem, [19, 10, 15], and opens the path to automatic calculations. Therefore, extending the Curci-Ferrari model to include quarks is the natural way to proceed in order to provide a tool similar to [14] for QCD. However, 
there is an additional practical point of view for our work. In conventional QCD perturbative calculations the fields of the Lagrangian are massless. However, in the real world the quarks have a physical mass. Therefore, including an explicit mass term for the quarks which is independent of the gluon mass we will have a Lagrangian which in principle includes as a special case the more realistic situation of massive quarks and massless gluons and ghosts as well as allowing us to interpolate between various different scenarios. For the two loop $\overline{\mathrm{MS}}$ renormalization of the Lagrangian we do not expect significant differences with conventional results for the renormalization group functions. However, the tool we provide here will, for example, be useful in studying the mixing of operators under renormalization to operators of the same and lower dimension since we will have an explicit quark mass at our disposal.

We take as our Lagrangian, [4, 6],

$$
\begin{aligned}
L= & -\frac{1}{4} G_{\mu \nu}^{a} G^{a \mu \nu}-\frac{1}{2 \alpha}\left(\partial^{\mu} A_{\mu}^{a}\right)^{2}+\frac{m^{2}}{2} A_{\mu}^{a} A^{a \mu}+\partial_{\mu} \bar{c}^{a} \partial^{\mu} c^{a}-\alpha m^{2} \bar{c}^{a} c^{a} \\
& -\frac{g}{2} f^{a b c} A_{\mu}^{a} \bar{c}^{b} \overleftrightarrow{\partial^{\mu}} c^{c}+\frac{\alpha g^{2}}{8} f^{e a b} f^{e c d} \bar{c}^{a} c^{b} \bar{c}^{c} c^{d} \\
& +i \bar{\psi}^{i I} \not \partial \psi^{i I}-\sqrt{\beta} m \bar{\psi}^{i I} \psi^{i I}-g \bar{\psi}^{i I} \gamma^{\mu} T_{I J}^{a} \psi^{i J} A_{\mu}^{a}
\end{aligned}
$$

where $1 \leq a \leq N_{A}, 1 \leq I \leq N_{F}$ with $N_{F}$ and $N_{A}$ the dimensions of the colour group fundamental and adjoint representations respectively where the structure constants are $f^{a b c}, 1 \leq i \leq N_{f}$ where $N_{f}$ is the number of quark flavours, $g$ is the coupling constant, $m$ is the gluon mass and hence the basic mass scale of the classical theory and $\bar{c}^{a} \overleftrightarrow{\partial_{\mu}} c^{b}=\bar{c}^{a} \partial_{\mu} c^{b}-\left(\partial_{\mu} \bar{c}^{a}\right) c^{b}$. The field strength, $G_{\mu \nu}^{a}$, follows from the definition of the covariant derivative as $G_{\mu \nu}^{a}=\partial_{\mu} A_{\nu}^{a}-\partial_{\nu} A_{\mu}^{a}-g f^{a b c} A_{\mu}^{b} A_{\nu}^{c}$. We have included the usual covariant gauge fixing term with parameter $\alpha$ which, to ensure that the action is BRST invariant, [4, 6, 8, 10], dictates the form of the ghost interactions with a gluon of mass $m$ and a ghost of mass $\sqrt{\alpha} m$. To make the two loop calculations easier to perform we have chosen to parameterize the quark mass with the parameter $\beta$ so that there is one basic mass parameter. This means that $\beta$ will get renormalized but the full quark mass, $\sqrt{\beta} m$, will be renormalized in such a way that it is independent of $\alpha$. The case of massive quarks but massless gluons is recovered by setting $m \rightarrow 0$ in such a way that $\sqrt{\beta} m$ remains finite. With this Lagrangian the Minkowski space propagators for the gluon, ghost and quark fields are respectively,

$$
-i \delta^{a b}\left[\frac{\eta^{\mu \nu}}{\left(k^{2}-m^{2}\right)}-\frac{(1-\alpha) k^{\mu} k^{\nu}}{\left(k^{2}-m^{2}\right)\left(k^{2}-\alpha m^{2}\right)}\right], \frac{i \delta^{a b}}{\left(k^{2}-\alpha m^{2}\right)} \quad, \frac{i \delta^{i j}(\not p-\sqrt{\beta} m)}{\left(p^{2}-\beta m^{2}\right)} .
$$

The renormalization of (1) proceeds along usual grounds. First, we introduce the renormalized variables via

$$
\begin{aligned}
A_{\mathrm{O}}^{a \mu} & =\sqrt{Z_{A}} A^{a \mu}, \quad c_{\mathrm{O}}^{a}=\sqrt{Z_{c}} c^{a}, \bar{c}_{\mathrm{o}}^{a}=\sqrt{Z_{c}} \bar{c}^{a}, \quad \psi_{\mathrm{o}}=\sqrt{Z_{\psi}} \psi \\
g_{\mathrm{o}} & =Z_{g} g, m_{\mathrm{O}}=Z_{m} m, \alpha_{\mathrm{o}}=Z_{\alpha}^{-1} Z_{A} \alpha, \quad \beta_{\mathrm{O}}=Z_{\beta} \beta
\end{aligned}
$$

where the subscript, o, denotes bare quantities. Since our calculation builds on the Yang-Mills version of the Curci-Ferrari model we have not assumed the usual Slavnov-Taylor identity of $Z_{\alpha}=1$. Therefore, we have seven independent renormalization constants to compute. However, we do have various cross-checks on the results we will obtain for the renormalization constants. Given that the parameter $m$ appears in both the gluon and ghost sectors we ought to obtain a consistent renormalization for it from considering independently the gluon and ghost two point functions. Moreover, we can check that our wave function renormalizations are correct by examining the various vertex corrections. The same coupling constant renormalization constant 
ought to emerge in all cases. Given the explicit values of the renormalization constants the corresponding renormalization group functions follow from the relations

$$
\begin{aligned}
\gamma_{A}(a) & =\beta(a) \frac{\partial \ln Z_{A}}{\partial a}+\alpha \gamma_{\alpha}(a) \frac{\partial \ln Z_{A}}{\partial \alpha} \\
\gamma_{\alpha}(a) & =\left[\beta(a) \frac{\partial \ln Z_{\alpha}}{\partial a}-\gamma_{A}(a)\right]\left[1-\alpha \frac{\partial \ln Z_{\alpha}}{\partial \alpha}\right]^{-1} \\
\gamma_{m}(a) & =-\beta(a) \frac{\partial \ln Z_{m}}{\partial a}-\alpha \gamma_{\alpha}(a) \frac{\partial \ln Z_{m}}{\partial \alpha} \\
\gamma_{\beta}(a) & =-\beta(a) \frac{\partial \ln Z_{\beta}}{\partial a}-\alpha \gamma_{\alpha}(a) \frac{\partial \ln Z_{\beta}}{\partial \alpha}
\end{aligned}
$$

where $\gamma_{\beta}(a)$ is defined by

$$
\gamma_{\beta}(a)=\frac{\mu}{\beta} \frac{\partial \beta}{\partial \mu}
$$

and $a=g^{2} /\left(16 \pi^{2}\right)$. (The parameter $\beta$ ought not to be confused with the $\beta$-function, $\beta(a)$.) In ordinary QCD one would have $\gamma_{A}(a)=-\gamma_{\alpha}(a)$ but since $Z_{\alpha} \neq 1$ in the Curci-Ferrari model we do not expect this to be restored in the presence of quarks. Moreover, this is the origin of the second terms in the expressions for $\gamma_{m}(a)$ and $\gamma_{\beta}(a)$ which will be dependent on $\alpha$.

Given that we are renormalizing a massive version of QCD we need to have an algorithm for computing the ultraviolet divergences of the massive multiscale two loop Feynman integrals which contribute. First, we use dimensional regularization with $d=4-2 \epsilon$. Second, we follow the strategy of [20, 21] where massive Feynman integrals are expanded in powers of the external momenta based on the identity

$$
\frac{1}{\left((k-p)^{2}-m^{2}\right)}=\frac{1}{\left(k^{2}-m^{2}\right)}+\frac{\left(2 k p-p^{2}\right)}{\left((k-p)^{2}-m^{2}\right)\left(k^{2}-m^{2}\right)} .
$$

The expansion is terminated by the rule that when the powers of momenta exceed those which can appear in the Green's function through renormalizability, then they are dropped. So, for example, the gluon propagator is only expanded to $O\left(p^{2}\right)$ with the $O\left(p^{3}\right)$ terms being dropped where $p$ is the external momentum. Consequently, one is left with massive vacuum bubble graphs where because of the different masses of (1), not all the masses are equal. However, massive vacuum two loop bubbles have been evaluated to the finite part in [22], for example, though we only require the Laurent expansion to the simple pole in $\epsilon$. For instance, the basic vacuum bubble with three different scales is given by

$$
\begin{aligned}
\int_{k l} \frac{1}{\left(k^{2}-m^{2}\right)\left(l^{2}-\alpha m^{2}\right)\left[(k-l)^{2}-\beta m^{2}\right]}=[ & -(1+\alpha+\beta)\left(\frac{1}{2 \epsilon^{2}}+\frac{3}{2 \epsilon}+\frac{1}{\epsilon} \ln \left(\frac{4 \pi}{m^{2} e^{\gamma}}\right)\right) \\
& \left.+(\alpha \ln \alpha+\beta \ln \beta) \frac{1}{\epsilon}+O(1)\right] \frac{m^{2}}{(4 \pi)^{4}} \quad(7)
\end{aligned}
$$

where $\gamma$ is the Euler-Mascheroni constant and $\int_{k}=\int d^{d} k /(2 \pi)^{d}$. The expression for different powers of the propagators are determined by differentiating with respect to the parameter $\alpha, \beta$ and $m^{2}$. It is worth noting that the appearance of $\ln \alpha$ and $\ln \beta$ terms could in principle lead to a non-analytic renormalization constant. However, these ought to cancel when all contributions from one and two loop graphs are included. In addition, in reducing the integrals to vacuum bubbles, partial fractions have been used which can give rise to other potentially singular terms. For instance,

$$
\frac{1}{\left(k^{2}-\alpha m^{2}\right)\left(k^{2}-\beta m^{2}\right)}=\frac{1}{(\alpha-\beta) m^{2}}\left[\frac{1}{\left(k^{2}-\alpha m^{2}\right)}-\frac{1}{\left(k^{2}-\beta m^{2}\right)}\right] .
$$


This provides another internal check since there are no singular terms in the original Lagrangian and for (1) to be renormalizable they ought not to remain after the two loop calculation. Given the large amount of algebra which arises due to the expansion to vacuum bubbles, to handle their evaluation for different masses we have written an algorithm in a symbolic manipulation language, Form version 3, [23]. The calculation proceeds automatically, since, for example the Feynman diagrams are generated using the QGRAF package, [24]. Moreover, the renormalization constants are extracted by computing the Green's functions in terms of bare parameters then rescaling by (3) after the pole structure has been determined and following the procedure of [25. This reproduces the usual method of subtractions automatically.

We have renormalized the gluon, ghost and quark two-point functions as well as the three 3 -point vertices. We obtain the following renormalization constants

$$
\begin{aligned}
& Z_{A}=1+\left[\left(\frac{13}{6}-\frac{\alpha}{2}\right) C_{A}-\frac{4}{3} T_{F} N_{f}\right] \frac{a}{\epsilon} \\
& +\left[\left(\left(\frac{3 \alpha^{2}}{16}-\frac{17 \alpha}{24}-\frac{13}{8}\right) C_{A}^{2}+C_{A} T_{F} N_{f}\left(\frac{2}{3} \alpha+1\right)\right) \frac{1}{\epsilon^{2}}\right. \\
& \left.-\left(\left(\frac{\alpha^{2}}{16}+\frac{11 \alpha}{16}-\frac{59}{16}\right) C_{A}^{2}+2 C_{F} T_{F} N_{f}+\frac{5}{2} C_{A} T_{F} N_{f}\right) \frac{1}{\epsilon}\right] a^{2}+O\left(a^{3}\right) \\
& Z_{\alpha}=1-\left(\frac{\alpha}{4}\right) C_{A} \frac{a}{\epsilon}+C_{A}^{2}\left[\left(\frac{\alpha^{2}}{16}+\frac{3 \alpha}{16}\right) \frac{1}{\epsilon^{2}}-\left(\frac{\alpha^{2}}{32}+\frac{5 \alpha}{32}\right) \frac{1}{\epsilon}\right] a^{2}+O\left(a^{3}\right) \\
& Z_{c}=1+\left(\frac{3}{4}-\frac{\alpha}{4}\right) C_{A} \frac{a}{\epsilon}+\left[\left(\left(\frac{\alpha^{2}}{16}-\frac{35}{32}\right) C_{A}^{2}+\frac{1}{2} C_{A} T_{F} N_{f}\right) \frac{1}{\epsilon^{2}}\right. \\
& \left.-\left(\left(\frac{\alpha^{2}}{32}-\frac{\alpha}{32}-\frac{95}{96}\right) C_{A}^{2}+\frac{5}{12} C_{A} T_{F} N_{f}\right) \frac{1}{\epsilon}\right] a^{2}+O\left(a^{3}\right) \\
& Z_{\psi}=1-\alpha C_{F} \frac{a}{\epsilon}+\left[\left(C_{F} C_{A}\left(\frac{\alpha^{2}}{8}+\frac{3 \alpha}{4}\right)+\frac{\alpha^{2}}{2} C_{F}^{2}\right) \frac{1}{\epsilon^{2}}\right. \\
& \left.-\left(C_{F} C_{A}\left(\alpha+\frac{25}{8}\right)-C_{F} T_{F} N_{f}-\frac{3}{4} C_{F}^{2}\right) \frac{1}{\epsilon}\right] a^{2}+O\left(a^{3}\right) \\
& Z_{m}=1+\left[\left(\frac{\alpha}{8}-\frac{35}{24}\right) C_{A}+\frac{2}{3} T_{F} N_{f}\right] \frac{a}{\epsilon} \\
& +\left[\left(\left(-\frac{\alpha^{2}}{128}-\frac{53 \alpha}{192}+\frac{1435}{384}\right) C_{A}^{2}+\frac{2}{3} T_{F}^{2} N_{f}^{2}+\left(\frac{\alpha}{12}-\frac{19}{6}\right) C_{A} T_{F} N_{f}\right) \frac{1}{\epsilon^{2}}\right. \\
& \left.+\left(\left(\frac{\alpha^{2}}{64}+\frac{11 \alpha}{64}-\frac{449}{192}\right) C_{A}^{2}+C_{F} T_{F} N_{f}+\frac{35}{24} C_{A} T_{F} N_{f}\right) \frac{1}{\epsilon}\right] a^{2}+O\left(a^{3}\right) \\
& Z_{\beta}=1+\left[\left(\frac{35}{12}-\frac{1}{\alpha}\right) C_{A}-\frac{4}{3} T_{F} N_{f}-6 C_{F}\right] \frac{a}{\epsilon} \\
& +\left[\left(\left(\frac{\alpha^{2}}{16}-\frac{13 \alpha}{24}-\frac{35}{32}\right) C_{A}^{2}+18 C_{F}^{2}+\left(\frac{\alpha}{3}+\frac{1}{2}\right) C_{A} T_{F} N_{f}\right.\right. \\
& \left.+4 C_{F} T_{F} N_{f}+\left(\frac{3 \alpha}{2}-\frac{13}{2}\right) C_{F} C_{A}\right) \frac{1}{\epsilon^{2}}-\left(\left(\frac{\alpha^{2}}{32}+\frac{11 \alpha}{32}-\frac{449}{96}\right) C_{A}^{2}\right. \\
& \left.\left.+\frac{3}{2} C_{F}^{2}-\frac{4}{3} C_{F} T_{F} N_{f}+\frac{35}{12} C_{A} T_{F} N_{f}+\frac{97}{6} C_{F} C_{A}\right) \frac{1}{\epsilon}\right] a^{2}+O\left(a^{3}\right) \\
& Z_{g}=1+\left(\frac{2}{3} T_{F} N_{f}-\frac{11}{6} C_{A}\right) \frac{a}{\epsilon}+\left[\left(\frac{121}{24} C_{A}^{2}+\frac{2}{3} T_{F}^{2} N_{f}^{2}-\frac{11}{3} C_{A} T_{F} N_{f}\right) \frac{1}{\epsilon^{2}}\right.
\end{aligned}
$$




$$
\left.+\left(C_{F} T_{F} N_{f}+\frac{5}{3} C_{A} T_{F} N_{f}-\frac{17}{6} C_{A}^{2}\right) \frac{1}{\epsilon}\right] a^{2}+O\left(a^{3}\right)
$$

where $T^{a} T^{a}=C_{F}, f^{a c d} f^{b c d}=C_{A} \delta^{a b}$ and $\operatorname{Tr}\left(T^{a} T^{b}\right)=T_{F} \delta^{a b}$. We have recorded these explicitly since the double pole in $\epsilon$ follows from the form of the one loop simple pole and therefore provides another check on our computation. Moreover, they are $\beta$-independent since we use a mass independent renormalization scheme. From these values we obtain the renormalization group functions at two loops in $\overline{\mathrm{MS}}$,

$$
\begin{aligned}
\gamma_{A}(a)= & {\left[(3 \alpha-13) C_{A}+8 T_{F} N_{f}\right] \frac{a}{6} } \\
& +\left[\left(\alpha^{2}+11 \alpha-59\right) C_{A}^{2}+40 C_{A} T_{F} N_{f}+32 C_{F} T_{F} N_{f}\right] \frac{a^{2}}{8}+O\left(a^{3}\right) \\
\gamma_{\alpha}(a)= & -\left[(3 \alpha-26) C_{A}+16 T_{F} N_{f}\right] \frac{a}{12} \\
& -\left[\left(\alpha^{2}+17 \alpha-118\right) C_{A}^{2}+80 C_{A} T_{F} N_{f}+64 C_{F} T_{F} N_{f}\right] \frac{a^{2}}{16}+O\left(a^{3}\right) \\
\gamma_{c}(a)= & (\alpha-3) C_{A} \frac{a}{4}+\left[\left(3 \alpha^{2}-3 \alpha-95\right) C_{A}^{2}+40 C_{A} T_{F} N_{f}\right] \frac{a^{2}}{48}+O\left(a^{3}\right) \\
\gamma_{\psi}(a)= & \alpha C_{F} a+C_{F}\left[(8 \alpha+25) C_{A}-6 C_{F}-8 T_{F} N_{f}\right] \frac{a^{2}}{4}+O\left(a^{3}\right) \\
\gamma_{m}(a)= & {\left[(3 \alpha-35) C_{A}+16 T_{F} N_{f}\right] \frac{a}{24} } \\
& +\left[\left(3 \alpha^{2}+33 \alpha-449\right) C_{A}^{2}+280 C_{A} T_{F} N_{f}+192 C_{F} T_{F} N_{f}\right] \frac{a^{2}}{96}+O\left(a^{3}\right) \\
& -\left[\left(3 \alpha^{2}+33 \alpha-449\right) C_{A}^{2}+1552 C_{F} C_{A}+280 C_{A} T_{F} N_{f}\right. \\
\gamma_{\beta}(a)= & \left.+144 C_{F}^{2}-128 C_{F} T_{F} N_{f}\right] \frac{a^{2}}{48}+O\left(a^{3}\right) \\
& -\left[\frac{11}{3} C_{A}-\frac{4}{3} T_{F} N_{f}\right] a^{2}-\left[\frac{34}{3} C_{A}^{2}-4 C_{F} T_{F} N_{f}-\frac{20}{3} C_{A} T_{F} N_{f}\right] a^{3}+O\left(a^{4}\right) . \\
\beta(a)= & \left.-72 C_{F}+16 T_{F} N_{f}\right] \frac{a}{12}
\end{aligned}
$$

The expression for the $\beta$-function agrees with the scheme independent results of [26, 27]. The renormalization group functions for the wave functions of the fields and $\alpha$ agree with the corresponding results of [25, 26, 27, 28] in the Landau gauge, $\alpha=0$. Indeed

$$
\gamma_{A}(a)+\gamma_{\alpha}(a)=\alpha\left[\frac{a}{4} C_{A}+(\alpha+5) C_{A}^{2} \frac{a^{2}}{16}\right]+O\left(a^{3}\right) .
$$

which is independent of $N_{f}$ and vanishes when $\alpha=0$. Moreover, (11) is equivalent to the statement that the ghost gluon vertex does not get renormalized in the Landau gauge, [29, 30], which was originally verified at one loop for (11) in [29]. With the presence of quarks the gluon mass dimension now depends on $N_{f}$ at one loop. However, to check we have correctly determined the quark mass anomalous dimension we need to compute the anomalous dimension of $m_{q}=\sqrt{\beta} m$. From

$$
\gamma_{m_{q}}(a)=\gamma_{m}(a)+\frac{1}{2} \gamma_{\beta}(a)
$$

we have

$$
\gamma_{m_{q}}(a)=-3 C_{F} a-C_{F}\left[97 C_{A}+9 C_{F}-20 T_{F} N_{f}\right] \frac{a^{2}}{6}+O\left(a^{3}\right)
$$


which agrees with the two loop $\overline{\mathrm{MS}}$ result of [31, 32] in our conventions and is independent of $\alpha$. For completeness, the ghost mass dimension is determined from

$$
m_{c}^{2}=\alpha m^{2}
$$

which implies

$$
\gamma_{m_{c}}(a)=\gamma_{m}(a)+\frac{1}{2} \gamma_{\alpha}(a)
$$

giving

$$
\gamma_{m_{c}}(a)=-\frac{3}{8} C_{A} a-\left[(18 \alpha+95) C_{A}^{2}-40 T_{F} N_{f}\right] \frac{a^{2}}{96}+O\left(a^{3}\right)
$$

which becomes $N_{f}$ and gauge dependent at two loops. Further checks on the correctness of $\gamma_{m}(a)$ are that the Yang-Mills sector of our expression agrees with the three loop result of [3] and the one loop expression of [33] where the multiplicative renormalizability of the composite operator $\frac{1}{2} A_{\mu}^{a} A^{a \mu}-\alpha \bar{c}^{a} c^{a}$ was verified by determining the mixing matrix of the renormalization of the constituent dimension two operators. For completeness, we have evaluated $\gamma(a)$ in the Landau gauge for QCD, $N_{c}=3$ and $T_{F}=\frac{1}{2}$, and found

$$
\left.\gamma_{m}(a)\right|_{\alpha=0}=\left(8 N_{f}-105\right) \frac{a}{24}+\left(548 N_{f}-4041\right) \frac{a^{2}}{96}+O\left(a^{3}\right) .
$$

It is worth noting that both coefficients are negative for $N_{f}<8$ which implies that the gluon mass runs to zero in the ultraviolet limit in this case.

In conclusion we have provided the full two loop renormalization of the Curci-Ferrari model with massive quarks in the $\overline{\mathrm{MS}}$ scheme. Whilst the nature of the model may appear unphysical with a gluon mass and lack of unitarity, it is important to recall that one long term aim is to use the the model to attack the renormalization of other Green's functions where quark mass effects will become important and a natural infrared mass regularization is necessary. Further, it is worth noting that models with non-abelian symmetries and massive gluons may have important phenomenological consequences. For instance, it has been shown in [33] that including $O\left(1 / Q^{2}\right)$ power corrections in the operator product expansion improves the matching of calculated physical quantities with experiment. Although such corrections derive from a gluon mass, albeit tachyonic in origin, they indicate the potential importance of massive gluons, such as that of (1), to probe non-perturbative physics.

\section{References.}

[1] K.-I. Kondo, Phys. Lett. B514 (2001), 335.

[2] K.-I. Kondo, hep-ph/0110013.

[3] H. Verschelde, K. Knecht, K. van Acoleyen \& M. Vanderkelen, Phys. Lett. B516 (2001), 307.

[4] G. Curci \& R. Ferrari, Nuovo Cim. A32 (1976), 151.

[5] G. Curci \& R. Ferrari, Nuovo Cim. A35 (1976), 1; G. Curci \& R. Ferrari, Nuovo Cim. A35 (1976), 273; Nuovo Cim. A47 (1978), 555.

[6] I. Ojima, Z. Phys. C13 (1982), 173.

[7] L. Baulieu, Phys. Rep. 13 (1985), 1; R. Delbourgo, S. Twisk \& G. Thompson, Int. J. Mod. Phys. A3 (1988), 435; T. Hurth, Helv. Phys. Acta 70 (1997), 406. 
[8] J. de Boer, K. Skenderis, P. van Nieuwenhuizen \& A. Waldron, Phys. Lett. B367 (1996), 175 .

[9] F. Delduc \& S.P. Sorella, Phys. Lett. B231 (1989), 408.

[10] A. Blasi \& N. Maggiore, Mod. Phys. Lett. A11 (1996), 1665.

[11] R. Delbourgo \& P.D. Jarvis, J. Phys. A15 (1982), 611.

[12] E. Andriyash \& K. Stepanyantz, hep-th/0204211.

[13] Ph. Boucaud, A. Le Yaounac, J.P. Leroy, J. Micheli, O. Pene \& J. Rodriguez-Quintero, Phys. Rev. D63 (2001), 114003.

[14] J.R. Forshaw, J. Papavassiliou \& C. Parrinello, Phys. Rev. D59 (1999), 074008.

[15] V. Periwal, hep-th/9509084.

[16] J.A. Gracey, Phys. Lett. B525 (2002), 89.

[17] A.A. Vladimirov, Theor. Math. Phys. 43 (1980), 417.

[18] K.G. Chetyrkin, A.L. Kataev \& F.V. Tkachov, Nucl. Phys. B174 (1980), 345.

[19] G. Curci \& E. d'Emilio, Phys. Lett. B83 (1979), 199.

[20] M. Misiak \& M. Münz, Phys. Lett. B344 (1995), 308.

[21] K.G. Chetyrkin, M. Misiak \& M. Münz, Nucl. Phys. B518 (1998), 473.

[22] C. Ford, I. Jack \& D.R.T. Jones, Nucl. Phys. B387 (1992), 373.

[23] J.A.M. Vermaseren, math-ph/0010025.

[24] P. Nogueira, J. Comput. Phys. 105 (1993), 279.

[25] S.A. Larin \& J.A.M. Vermaseren, Phys. Lett. B303 (1993), 334.

[26] D.J. Gross \& F.J. Wilczek, Phys. Rev. Lett. 30 (1973), 1343; H.D. Politzer, Phys. Rev. Lett. 30 (1973), 1346.

[27] D.R.T. Jones, Nucl. Phys. B75 (1974), 531; W.E. Caswell, Phys. Rev. Lett. 33 (1974), 244.

[28] O.V. Tarasov \& A.A. Vladimirov, Sov. J. Nucl. Phys. 25 (1977), 585; E. Egorian \& O.V. Tarasov, Theor. Math. Phys. 41 (1979), 863.

[29] L. Baulieu \& J. Thierry-Mieg, Nucl. Phys. B197 (1982), 477.

[30] R. Alkofer \& L. von Smekal, Phys. Rept. 353 (2001), 281.

[31] D.V. Nanopoulos \& D.A. Ross, Nucl. Phys. B157 (1979), 273.

[32] R. Tarrach, Nucl. Phys. B183 (1981), 384; O. Nachtmann \& W. Wetzel, Nucl. Phys. B187 (1981), 333.

[33] K.-I. Kondo, T. Murakami, T. Shinohara \& T. Imai, Phys. Rev. D65 (2002), 085034.

[34] K.G. Chetyrkin, S. Narison \& V.I. Zakharov, Nucl. Phys. B550 (1999), 353. 